\title{
Response to Letter to the Editor Re: The Effect of PPI Use on Human Gut Microbiota and Weight Loss in Patients Undergoing Laparoscopic Roux-en-Y Gastric Bypass
}

\author{
Gregory L. Austin ${ }^{1}$ - Emily K. Ward ${ }^{1}$
}

Published online: 10 April 2015

(C) Springer Science+Business Media New York 2015

To the Editor:

We appreciate the interest and comments of Syu, Huang, and Chen on our article regarding the effect of PPI use on gut microbiome profiles and weight loss in patients undergoing laparoscopic Roux-en-Y gastric bypass (LRYGB) [1]. We also greatly appreciate their report on their own experience in evaluating the association of short-term PPI use with weight gain. Given that patients who take a PPI for gastroesophageal reflux disease are more likely to be obese, the issue of whether PPI use is associated with weight gain or impaired weight loss is an important question.

The data presented by Syu et al. are interesting and an important contribution to the literature in which there is a paucity of data. Of note, the population studied by Syu et al. was considerably different compared to the bariatric surgery population included in our study. First, their cohort included only non-obese patients who had endoscopically proven Helicobacter pylori-negative gastric ulcers. Second, the gut microbiota was not studied in Syu's cohort, but it is an important distinguishing characteristic that may explain how PPI use influences weight change. Non-obese patients have been shown to have a different gut microbiota profile compared to obese patients [2]. Our study indicated that PPI users had an

Gregory L. Austin

gregory.austin@ucdenver.edu

1 Division of Gastroenterology and Hepatology, University of Colorado Denver, 12631 East 17th Avenue, Room 7619, Aurora, CO 80045, USA

altered gut microbiome composition both before and after LRYGB that was associated with impaired weight loss. Finally, the mean age of the cohort of Syu et al. was considerably older ( 63.8 versus 44.3 years), and the proportion of females ( 29 versus $88 \%$ ) was lower compared to the patients in our study. In a separate cohort, we recently reported on the association of PPI use with suboptimal weight loss in 472 consecutive patients who underwent LRYGB, a cohort whose demographics are also significantly different than those included in the study by Syu et al. [3].

The effect of PPI use on body weight may vary significantly across groups with different characteristics and may depend upon an individual's underlying propensity for obesity, baseline gut microbiota, as well as their age or gender. We are exploring mechanisms that might explain how PPI use leads to weight gain. Given the high prevalence of PPI use, addressing these unresolved questions is of critical importance.

Conflict of Interest No conflicts of interest exist for any of the authors. No writing assistance was provided for this manuscript.

\section{References}

1. Ward EK, Schuster DP, Stowers KH, et al. The effect of PPI use on human Gut microbiota and weight loss in patients undergoing laparoscopic Roux-en-Y gastric bypass. Obes Surg. 2014;24(9):1567-71. doi:10.007/s11695-014-1275-1.

2. Turnbaugh PJ, Hamady M, Yatsunenko T, et al. A core gut microbiome in obese and lean twins. Nature. 2009;457(7228):480-4.

3. Ward EK, Jensen-Otsu E, Schoen JA, et al. Acid suppression medications are associated with suboptimal weight loss after laparoscopic Roux-en-Y gastric bypass in patients older than 40 years. Surg Obes Relat Dis. 2014;7(14):00369-4. 